\title{
Quantitative image variables reflect the intratumoral pathologic heterogeneity of lung adenocarcinoma
}

\author{
E-Ryung Choi ${ }^{1}$, Ho Yun Lee ${ }^{1}$, Ji Yun Jeong ${ }^{2}$, Yoon-La Choi ${ }^{3}$, Jhingook Kim ${ }^{4}$, Jungmin \\ Bae $^{1}$, Kyung Soo Lee ${ }^{1}$, Young Mog Shim ${ }^{4}$ \\ ${ }^{1}$ Department of Radiology and Center for Imaging Science, Samsung Medical Center, Sungkyunkwan University School of \\ Medicine, Seoul, Korea \\ ${ }^{2}$ Department of Pathology, Kyungpook National University Hospital, Kyungpook National University School of Medicine, \\ Daegu, Korea \\ ${ }^{3}$ Department of Pathology, Samsung Medical Center, Sungkyunkwan University School of Medicine, Seoul, Korea \\ ${ }^{4}$ Department of Thoracic and Cardiovascular Surgery, Samsung Medical Center, Sungkyunkwan University School of Medicine, \\ Seoul, Korea
}

Correspondence to: Ho Yun Lee, email: hoyunlee96@gmail.com

Keywords: Iung adenocarcinoma, heterogeneity, radiomics, quantitative image variables, dual energy CT

Received: May 12, $2016 \quad$ Accepted: July 19, $2016 \quad$ Published: August 30, 2016

\section{ABSTRACT}

We aimed to compare quantitative radiomic parameters from dual-energy computed tomography (DECT) of lung adenocarcinoma and pathologic complexity.

A total 89 tumors with clinical stage I/II lung adenocarcinoma were prospectively included. Fifty one radiomic features were assessed both from iodine images and noncontrast images of DECT datasets. Comprehensive histologic subtyping was evaluated with all surgically resected tumors. The degree of pathologic heterogeneity was assessed using pathologic index and the number of mixture histologic subtypes in a tumor. Radiomic parameters were correlated with pathologic index. Tumors were classified as three groups according to the number of mixture histologic subtypes and radiomic parameters were compared between the three groups.

Tumor density and $50^{\text {th }}$ through $97.5^{\text {th }}$ percentile Hounsfield units (HU) of histogram on non-contrast images showed strong correlation with the pathologic heterogeneity. Radiomic parameters including $75^{\text {th }}$ and $97.5^{\text {th }}$ percentile $\mathrm{HU}$ of histogram, entropy, and inertia on 1-, 2- and 3 voxel distance on non-contrast images showed incremental changes while homogeneity showed detrimental change according to the number of mixture histologic subtypes (all Ps $<0.05$ ).

Radiomic variables from DECT of lung adenocarcinoma reflect pathologic intratumoral heterogeneity, which may help in the prediction of intratumoral heterogeneity of the whole tumor.

\section{INTRODUCTION}

Lung cancer is the most commonly diagnosed cancer worldwide and the leading cause of cancer-related death [1], and adenocarcinoma is the most common histologic subtype of lung cancer in most countries [2]. In an attempt to reflect the widely divergent pathologic spectrum of lung adenocarcinoma, lung adenocarcinoma classification criteria were proposed by the International Association for the Study of Lung Cancer/American Thoracic Society/European Respiratory Society (IASLC/ATS/ ERS) [3]. This classification was devised to understand the histological subtypes and their histo-molecular correlations. Since the release of this classification, many studies have investigated the possible correlations among the most predominant subtypes, driver mutations and patient prognosis $[4,5]$. However, prognostic stratification considering only the most predominant subtypes has shown substantial limitation due to the fact that more than $80 \%$ of invasive lung adenocarcinomas show mixed type including two or more of histologic subtypes [6-9].

Recently, scientists have performed quantitative imaging of lung cancer using primarily a radiomic approach, demonstrating that radiomic values quantifying 
spatial variation in architecture have shown prognostic significance [10-12]. Based on these results, they suggested that imaging features depicting spatial heterogeneity in tumors might reflect genomic and phenotypic intratumoral heterogeneity, which has significant implications for treatment, resistance, and, ultimately, prognosis [13-15]. Surprisingly, there have not been any studies directly evaluating the relationship between radiologic heterogeneity values and pathologic complexity within lung adenocarcinoma. Thus, we aimed to correlate various quantitative radiomic parameters from dual-energy computed tomography (DECT) of lung adenocarcinomas with pathologic complexity to ultimately identify the role of quantitative image variables in predicting pathologic heterogeneity.

\section{RESULTS}

\section{Clinical characteristics of patients and tumors}

All 92 consecutive patients were enrolled and underwent DECT for work-up (Figure 1). We excluded three patients who were shown to have benign disease after percutaneous lesion biopsy and five patients who were found to have unresectable stage III or IV lung cancer through further studies. Eight-four patients underwent complete resection for ninety-three lesions. Of those, we excluded four who had one benign disease, one who had mucinous adenocarcinoma, and two who had insufficient pathologic slides for detailed pathologic review. In total, 80 patients with 89 lung adenocarcinomas were included in our study.

There were total 6 patients who have more than one primary lesion arising in the lung at the same time. Three patients had 2 tumors in the lung and three patients had 3 tumors in the lung. Among these patients, four had nodules in the same lobe. However, multiple tumors in the same lobe showed complete different solidity on CT scan suggesting intendent relationships more likely than the metastasis. Pathological result revealed these tumors were synchronous tumors. The most predominant histologic subtype was the acinar subtype (59.6\%) followed by lepidic subtype (20.2\%) (Table 1$)$. The most frequent combinations in mixed type were lepidic and acinar subtypes (14.6\%). Final pathologic staging revealed 84 stage I tumors, one stage II tumor, and four stage III tumors. The clinicopathologic characteristics of the 89 lung adenocarcinomas included in this study are summarized in Table 1. Also, the relationship between size and volume of tumors are included in Supplementary Figure S1.

\section{Correlation between radiomic features and pathology heterogeneity index}

There was strong relationship between the prediction model made with selected radiomic variables and pathologic heterogeneity index $(R=0.936, p=0.001)$.

The relationships between 51 radiomic parameters and the pathologic heterogeneity index are described

\section{Dual energy CT underwent for \\ suspected early stage lung adenocarcinoma}

(Stage I or II) $(n=101)$

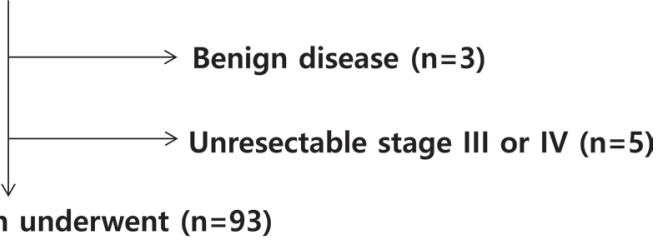

Complete resection underwent $(n=93)$
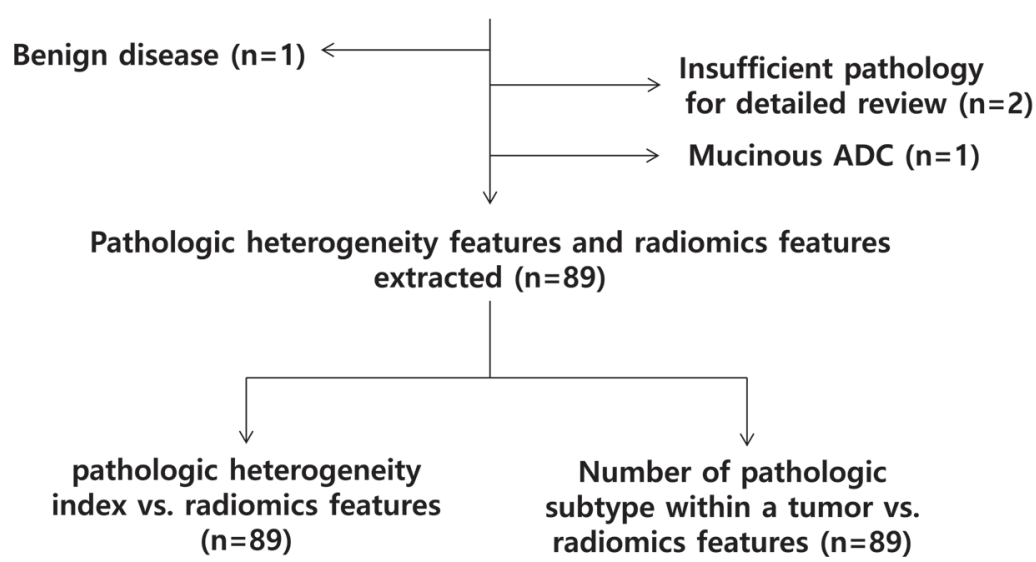

Figure 1: Flow diagram of the patient cohort. 
in Table 2. Among the radiomic parameters of physical features, density on non-contrast images showed a strong correlation with pathologic heterogeneity $(R=$ $0.654, P<0.001)$. Also, pathologic heterogeneity was robustly associated with $50^{\text {th }}$ percentile, $75^{\text {th }}$ percentile and $97.5^{\text {th }}$ percentile of HU among histogram CT parameters on non-contrast images $(R=0.648, R=$ $0.663, R=0.626$, respectively; all $P s<0.001)$. The size of the tumor on iodine-contrast images shows moderate positive correlation $(R=0.527, P<0.001)$. The skewness of histogram features showed a moderate negative correlation with histologic complexity on both non-contrast and iodine-contrast images $(R=-0.532$, $R=-0.531$, respectively; all $P S<0.001)$. There was no strong association between regional features. Among local features, variance and inertia on non-contrast image were moderately associated with pathologic complexity (variance on 1 voxel distance, $R=-0.522$; variance on 2 voxel distance, $R=-0.517$; variance on 3 voxel distance, $R=-0.521$; inertia on 1 voxel distance, $R=0.549$; inertia on 2 voxel distance, $R=0.550$; inertia on 3 voxel distance, $R=0.574 ;$ all $\left.P_{S}<0.001\right)$.

\section{Comparison of three different groups based on pathologic heterogeneity and radiomic parameters}

Among histogram features on non-contrast CT images, the $75^{\text {th }}$ percentile and $97.5^{\text {th }}$ percentile HU of the histogram showed significantly increased mean $\mathrm{CT}$ values according to the number of histologic subtypes $(P=0.003$, $P=0.002$, respectively) (Table 3 ).

Of all regional features, entropy showed an escalation of the mean $\mathrm{CT}$ values with an increased number of histologic subtypes on non-contrast images with statistical significance $(P=0.003)$. Although those were not statistically significant, intensity variability and size-zone variability on the non-contrast images and entropy on the iodine images presented increased mean CT values according to the number of histologic subtypes.

Among local features on non-contrast images, the mean CT values of inertia increased while those of homogeneity demonstrate a declined as the number of histologic subtypes increased with statistical significance (Inertia on 1 voxel distance, $P=0.027$; Inertia on 2 voxel distance, $P=0.024$; Inertia on 3 voxel distance, $P=0.018$; homogeneity on 1 voxel distance, $P=025$; homogeneity on 2 voxel distance, $P=023$; homogeneity on 3 voxel distance, $P=0.008)$. On iodine-enhanced images, the mean CT values of energy and maximum probability value increased while those of contrast, sum mean, cluster shade, and homogeneity showed a decline when the number of histologic subtypes increased. However, the tendencies of these values were failed to show the statistical significance.
All radiomic parameters of the three different groups based on pathologic heterogeneity are described in Table 3.

\section{DISCUSSION}

Recent lung cancer research has demonstrated that cancerous cells not only undergo clonal evolution from a single progenitor, but also exhibit branched evolution, whereby each tumor develops and preserves multiple distinct subclonal compositions [16-19]. This intratumoral genetic heterogeneity consequently leads to phenotypic differences in histopathological divergence, containing regions demarcated by various degrees of differentiation, proliferation, vascularity, and invasiveness [20]. Given the existence of such heterogeneity in tumors in advanced metastatic disease, the efficacy of therapies targeting somatic driver aberrations may be limited [13]. Recent oncology research has mostly focused on the intratumoral variation in gene mutation and expression, whereas few studies have explored the spatial relationship among imaging, genomics, and histopathology.

While imaging is a main tool in tumor staging, progression assessment and recurrence detection, most radiologic approaches deal only with tumor size or average parameter values [12] in routine oncologic practice and research. Considerable effort has explored sophisticated and robust analyses to quantify tumor spatial complexity with tumor imaging data [10, 21-25]. This approach usually uses a texture analysis method to quantify the spatial variation as the remaining spatial arrangement of voxel values in imaging data [12], which could serve as a potential prognostic biomarker $[11,26]$. Most studies of radiomic analyses have evaluated the role of radiomics in the prognostic stratification [12], but there have not yet been any studies correlating intratumoral heterogeneity on CT images with pathology heterogeneity. Radiomic analysis is emerging as a method to quantify spatial variation within tumors. Establishment of a histologic correlation would obviously be an important step in the validation of this approach. Our study is the first study to determine a direct relationship between the histological complexity of lung adenocarcinoma and intratumoral heterogeneity expressed as CT radiomic values.

We adopted histogram, local and regional features to measuring degree of intratumoral heterogeneity. Histogram features were derived from the density distribution of the tumor reflecting heterogeneity of intratumoral density. The spatial arrangement of voxel values is obtained from local features. We included tumor size as a routine practical measurement variable and compared with other all radiomic variables in the analysis. Radiomic variables were more strongly correlated with pathologic heterogeneity rather than tumor size. In 
Table 1: Clinicopathologic characteristics of lung adenocarcinoma (89 tumors from 80 patients)

\begin{tabular}{|c|c|c|}
\hline Characteristics & \multicolumn{2}{|l|}{ Total N (\%) } \\
\hline \multicolumn{3}{|l|}{$\operatorname{Sex}(\%)$} \\
\hline Male & \multicolumn{2}{|l|}{$39(48.8)$} \\
\hline Female & \multicolumn{2}{|l|}{$41(51.2)$} \\
\hline Age, range (median) & \multicolumn{2}{|l|}{$37-78(59)$} \\
\hline $\begin{array}{l}\text { Tumor size }(\mathrm{mm}), \\
\text { range (median) }\end{array}$ & \multicolumn{2}{|l|}{$4-64(21)$} \\
\hline \multicolumn{3}{|l|}{ Staging } \\
\hline I & \multicolumn{2}{|l|}{$84(94.4)$} \\
\hline II & \multicolumn{2}{|l|}{$1(1.1)$} \\
\hline III & \multicolumn{2}{|l|}{$4(4.5)$} \\
\hline \multicolumn{3}{|l|}{$\mathbf{T}$ category } \\
\hline 1 & \multicolumn{2}{|l|}{$73(82.1)$} \\
\hline 2 & \multicolumn{2}{|l|}{$14(15.7)$} \\
\hline 3 & \multicolumn{2}{|l|}{$2(2.2)$} \\
\hline \multicolumn{3}{|l|}{ N category } \\
\hline 0 & \multicolumn{2}{|l|}{$82(92.1)$} \\
\hline 1 & $5(5.6)$ & \\
\hline 2 & $2(2.2)$ & \\
\hline Extent of resection $(\%$ & & \\
\hline Segmentectomy & $50(56.2)$ & \\
\hline Lobectomy & $39(43.8)$ & \\
\hline $\begin{array}{l}\text { Most predominant } \\
\text { histologic subtype }\end{array}$ & & \\
\hline Lepidic & $18(20.2)$ & \\
\hline Acinar & $53(59.6)$ & \\
\hline Papillary & $11(12.4)$ & \\
\hline Solid & $1(1.1)$ & \\
\hline Micropapillary & $6(6.7)$ & \\
\hline No. of histologic subt & thin a tumor & \\
\hline $19(21.3)$ & Lepidic & $5(26.3)$ \\
\hline & Acinar & $11(57.9)$ \\
\hline & Papillary & $3(15.8)$ \\
\hline $54(60.7)$ & Lepidic + Acinar & $31(57.4)$ \\
\hline & Acinar + Papillary & $13(24.1)$ \\
\hline & Acinar + Micropapillary & $1(1.9)$ \\
\hline & Acinar + Solid & $9(16.7)$ \\
\hline $14(15.7)$ & Lepidic + Acinar + Papillary & $6(42.9)$ \\
\hline & Lepidic + Acinar + Solid & $2(14.3)$ \\
\hline & Acinar + Papillary + Micropapillary & $4(28.6)$ \\
\hline & Acinar + Micropapillary + Solid & $2(14.3)$ \\
\hline $2(2.2)$ & Lepidic + Acinar + Papillary + Micropapillary & $2(100)$ \\
\hline
\end{tabular}


Table 2: Correlation of radiomic features with pathologic heterogeneity index

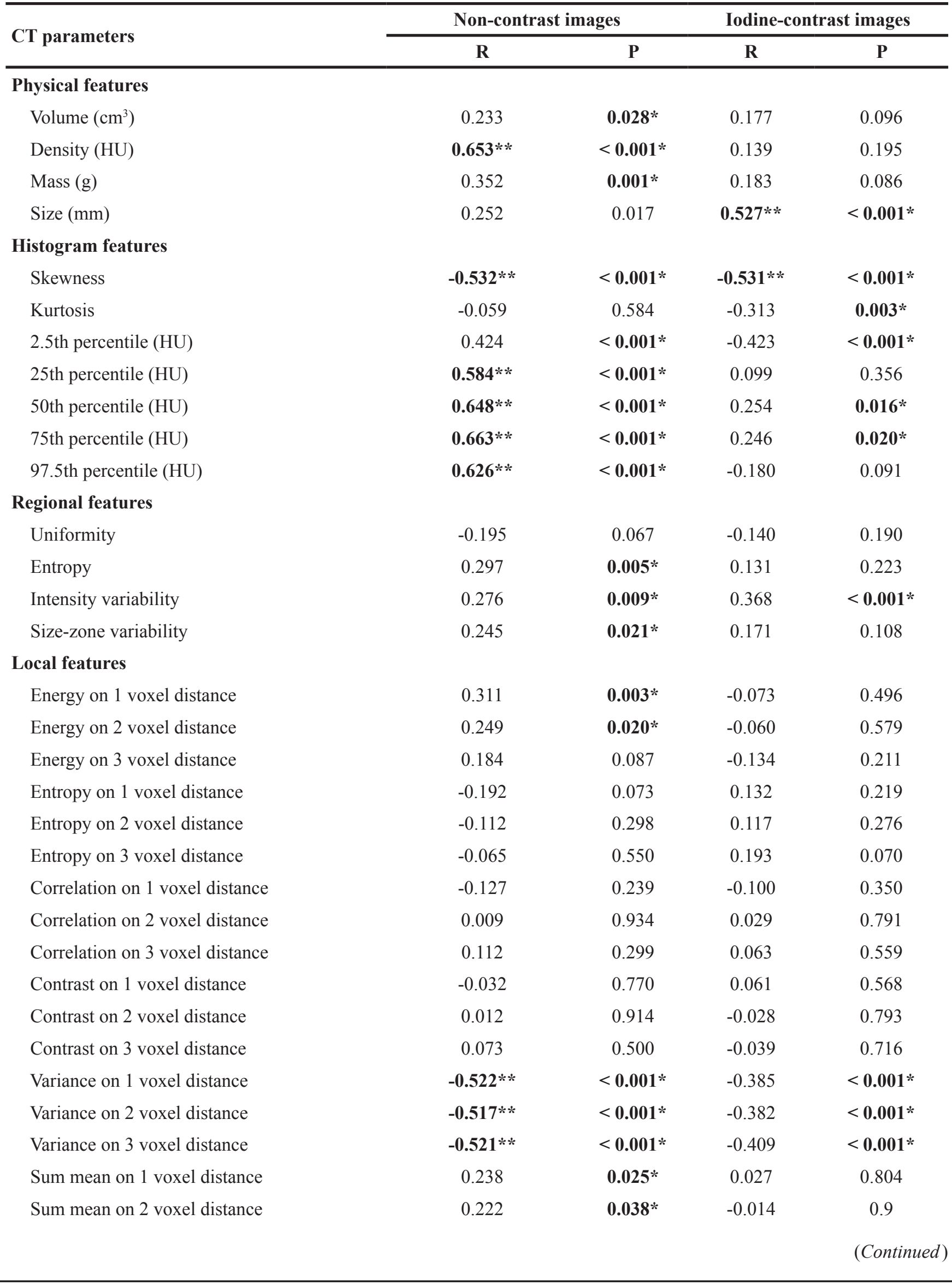




\begin{tabular}{|c|c|c|c|c|}
\hline \multirow{2}{*}{ CT parameters } & \multicolumn{2}{|c|}{ Non-contrast images } & \multicolumn{2}{|c|}{ Iodine-contrast images } \\
\hline & $\mathbf{R}$ & $\mathbf{P}$ & $\mathbf{R}$ & $\mathbf{P}$ \\
\hline Sum mean on 3 voxel distance & 0.235 & $0.027 *$ & -0.017 & 0.875 \\
\hline Inertia on 1 voxel distance & $0.549 * *$ & $<0.001 *$ & 0.391 & $<0.001 *$ \\
\hline Inertia on 2 voxel distance & $0.550 * *$ & $<0.001 *$ & 0.384 & $<0.001 *$ \\
\hline Inertia on 3 voxel distance & $0.574 * *$ & $<0.001 *$ & 0.401 & $<0.001 *$ \\
\hline Cluster shade on 1 voxel distance & -0.032 & 0.770 & 0.061 & 0.568 \\
\hline Cluster shade on 2 voxel distance & 0.012 & 0.914 & -0.028 & 0.793 \\
\hline Cluster shade on 3 voxel distance & 0.073 & 0.500 & -0.039 & 0.716 \\
\hline Cluster tendency on 1 voxel distance & -0.454 & $<0.001 *$ & -0.434 & $<0.001 *$ \\
\hline Cluster tendency on 2 voxel distance & -0.438 & $<0.001 *$ & -0.420 & $<0.001 \%$ \\
\hline Cluster tendency on 3 voxel distance & -0.448 & $<0.001 *$ & -0.470 & $<0.001 *$ \\
\hline Homogeneity on 1 voxel distance & 0.406 & $<0.001 *$ & -0.207 & 0.052 \\
\hline Homogeneity on 2 voxel distance & 0.398 & $<0.001 *$ & -0.129 & 0.230 \\
\hline Homogeneity on 3 voxel distance & 0.411 & $<0.001 *$ & -0.109 & 0.308 \\
\hline Maximum probability on 1 voxel distance & 0.447 & $<0.001 *$ & -0.041 & 0.700 \\
\hline Maximum probability on 2 voxel distance & 0.403 & $<0.001 *$ & -0.042 & 0.698 \\
\hline Maximum probability on 3 voxel distance & 0.323 & $0.002 *$ & -0.090 & 0.400 \\
\hline Inverse variance on 1 voxel distance & -0.336 & $0.001 *$ & -0.102 & 0.343 \\
\hline Inverse variance on 2 voxel distance & -0.124 & 0.250 & -0.005 & 0.963 \\
\hline Inverse variance on 3 voxel distance & -0.048 & 0.659 & -0.023 & 0.833 \\
\hline
\end{tabular}

$\mathrm{R}$ indicates the Spearman correlation coefficient. $* \mathrm{P}<0.05, * * \mathrm{R}>0.5$

addition, these radiomic features may offer minimal interand intra-reader variability with high reproducibility of imaging features.

Our results clearly demonstrate histopathologic complexity correlated with radiomic parameters such as texture features within $\mathrm{CT}$ images of lung adenocarcinoma. Quantifying the spatial complexity of tumor images can explain the spatial complexity in pathology, or in other words, the phenotypic intratumoral heterogeneity.

Radiomic features derived from non-contrast images reflect tumor cellularity and density, whereas iodineenhanced image even allows additional information about tumor vascularity. Therefore, we originally expected that radiomic features of iodine-contrast images might have associated more strongly to the tumor complexity related with heterogeneity of tumor density and tumor vascularity. However, in our analysis, the most of radiomic features which correlated to pathologic heterogeneity index were features derived from non-contrast image, not from iodine-enhanced image. This result can be explained in part by the concept of tumor microenvironment where whole tumor volume consists of real tumor component as well as nontumorous stromal component. Thus, radiomic variables extracted from non-contrast $\mathrm{CT}$ covering completely density or cellularity of ROI of the tumor may reflect more actually spatial heterogeneity in the entire tumor ROI, compared to radiomic variables from iodine image limitedly enhancing heterogeneity of only tumorous component. The problem is that results from small biopsy tissues in non-resectable lung adenocarcinomas do not represent the pathologic heterogeneity of the whole tumor. On the other hand, quantifying imaging data using a radiomic approach indirectly enables assessment of whole tumor heterogeneity. Quantifying spatial heterogeneity on a tumor image may help with determining the prognosis and stratifying patients with non-resectable lung adenocarcinoma for which whole tumor pathology is not available. Furthermore, intratumoral heterogeneity may have important consequences for personalized medicine approaches that typically rely on a single tumor-biopsy to portray the tumor mutational landscape. Quantitative radiomic variables could allow physicians to deliver more optimized, patient-specific treatment by allowing them to identify patients who need aggressive treatment. 
Table 3: Radiomic features according to the number of histologic subtypes within a tumor

\begin{tabular}{|c|c|c|c|c|c|c|c|c|}
\hline & \multicolumn{3}{|c|}{ Non-contrast images } & \multirow[b]{2}{*}{$p$} & \multicolumn{3}{|c|}{ Iodine-contrast images } & \multirow[b]{2}{*}{$p$} \\
\hline & One subtype & Two subtypes & $\begin{array}{c}\text { Three or four } \\
\text { subtypes }\end{array}$ & & One subtype & Two subtypes & $\begin{array}{l}\text { Three or four } \\
\text { subtypes }\end{array}$ & \\
\hline \multicolumn{9}{|l|}{ Physical features } \\
\hline Volume $\left(\mathrm{cm}^{3}\right)$ & $6.97 \pm 2.14$ & $8.29 \pm 2.81$ & $7.93 \pm 5.900$ & 0.966 & $7.08 \pm 12.62$ & $4.67 \pm 5.85$ & $6.50 \pm 5.10$ & 0.432 \\
\hline Density (HU) & $0.57 \pm 0.23$ & $0.566 \pm 0.22$ & $0.75 \pm 0.155$ & $0.008 *$ & $1.17 \pm 0.016$ & $1.16 \pm 0.019$ & $1.17 \pm 0.01$ & 0.618 \\
\hline Mass (g) & $5.30 \pm 10.35$ & $4.572 \pm 9.00$ & $6.00 \pm 4.484$ & 0.832 & $8.25 \pm 14.71$ & $5.44 \pm 6.78$ & $7.61 \pm 5.95$ & 0.426 \\
\hline Size (mm) & $23.11 \pm 16.86$ & $21.02 \pm 9.26$ & $24.41 \pm 10.89$ & 0.488 & $12.44 \pm 18.26$ & $9.83 \pm 12.02$ & $17.18 \pm 8.85$ & 0.130 \\
\hline \multicolumn{9}{|l|}{ Histogram features } \\
\hline Skewness & $-0.21 \pm 0.66$ & $0.012 \pm 0.721$ & $-0.489 \pm 0.616$ & $0.037 *$ & $0.461 \pm 0.822$ & $0.782 \pm 1.04$ & $0.056 \pm 0.598$ & 0.024 * \\
\hline Kurtosis & $2.75 \pm 0.96$ & $2.76 \pm 1.01$ & $2.47 \pm 1.108$ & 0.598 & $4.39 \pm 2.32$ & $5.60 \pm 4.33$ & $4.51 \pm 3.29$ & 0.377 \\
\hline $2.5^{\text {th }}$ percentile $(\mathrm{HU})$ & $-797.76 \pm 71.95$ & $-814.99 \pm 70.14$ & $-769.28 \pm 74.37$ & 0.080 & $-21.07 \pm 14.38$ & $-23.42 \pm 16.68$ & $-31.39 \pm 14.34$ & 0.131 \\
\hline $25^{\text {th }}$ percentile $(\mathrm{HU})$ & $-576.08 \pm 193.86$ & $-604.91 \pm 181.72$ & $-484.64 \pm 181.10$ & 0.078 & $25.31 \pm 13.29$ & $21.15 \pm 16.143$ & $26.20 \pm 15.66$ & 0.397 \\
\hline $50^{\text {th }}$ percentile $(\mathrm{HU})$ & $-431.21 \pm 232.06$ & $-450.78 \pm 234.06$ & $-251.37 \pm 171.56$ & $0.009 *$ & $52.50 \pm 16.20$ & $47.94 \pm 19.68$ & $56.62 \pm 5.35$ & 0.220 \\
\hline $75^{\text {th }}$ percentile $(\mathrm{HU})$ & $-316.05 \pm 241.84$ & $-305.18 \pm 232.75$ & $-91.90 \pm 127.34$ & $0.003 *$ & $79.94 \pm 17.36$ & $76.38 \pm 22.41$ & $83.68 \pm 16.05$ & 0.429 \\
\hline $97.5^{\text {th }}$ percentile $(\mathrm{HU})$ & $-181.87 \pm 241.84$ & $-128.25 \pm 189.22$ & $32.48 \pm 39.52$ & $0.002 *$ & $152.31 \pm 39.92$ & $156.08 \pm 35.30$ & $143.65 \pm 27.89$ & 0.464 \\
\hline \multicolumn{9}{|l|}{ Regional features } \\
\hline Uniformity & $0.003 \pm 0.002$ & $0.002 \pm 0.001$ & $0.002 \pm 0.001$ & $0.042 *$ & $0.009 \pm 0.002$ & $0.009 \pm 0.002$ & $0.008 \pm 0.002$ & 0.365 \\
\hline Entropy & $8.76 \pm 0.73$ & $9.08 \pm 0.50$ & $9.41 \pm 0.37$ & $0.003 *$ & $7.17 \pm 0.37$ & $7.24 \pm 0.32$ & $7.36 \pm 0.31$ & 0.258 \\
\hline Intensity variability & $7.56 \pm 3.71$ & $8.49 \pm 3.8$ & $9.57 \pm 3.53$ & 0.294 & $7.27 \pm 3.18$ & 6.1563 .29 & $8.03 \pm 3.27$ & 0.099 \\
\hline Size-zone variability & $13.14 \pm 12.21$ & $15.33 \pm 11.86$ & $18.59 \pm 7.81$ & 0.367 & $14.42 \pm 14.94$ & $14.22 \pm 9.44$ & $17.99 \pm 8.60$ & 0.454 \\
\hline \multicolumn{9}{|l|}{ Local features } \\
\hline Energy on 1 voxel distance & $0.040 \pm 0.050$ & $0.034 \pm 0.033$ & $0.051 \pm 0.067$ & 0.410 & $0.037 \pm 0.017$ & $0.046 \pm 0.030$ & $0.049 \pm 0.036$ & 0.394 \\
\hline Energy on 2 voxel distance & $0.036 \pm 0.046$ & $0.027 \pm 0.028$ & $0.042 \pm 0.064$ & 0.356 & $0.033 \pm 0.014$ & $0.040 \pm 0.025$ & $0.043 \pm 0.033$ & 0.493 \\
\hline Energy on 3 voxel distance & $0.031 \pm 0.025$ & $0.024 \pm 0.026$ & $0.039 \pm 0.061$ & 0.337 & $0.036 \pm 0.014$ & $0.040 \pm 0.025$ & $0.042 \pm 0.033$ & 0.784 \\
\hline Entropy on 1 voxel distance & $1.74 \pm 0.23$ & $1.79 \pm 0.19$ & $1.76 \pm 0.24$ & 0.580 & $1.65 \pm 0.15$ & $1.62 \pm 0.18$ & $1.59 \pm 0.22$ & 0.697 \\
\hline Entropy on 2 voxel distance & $1.82 \pm 0.24$ & $1.91 \pm 0.20$ & $1.89 \pm 0.26$ & 0.306 & $1.66 \pm 0.13$ & $1.66 \pm 0.17$ & $1.63 \pm 0.22$ & 0.869 \\
\hline Entropy on 3 voxel distance & $1.81 \pm 0.22$ & $1.93 \pm 0.22$ & $1.92 \pm 0.28$ & 0.128 & $1.62 \pm 0.15$ & $1.65 \pm 0.16$ & $1.63 \pm 0.22$ & 0.798 \\
\hline Correlation on 1 voxel distance & $0.089 \pm 0.037$ & $0.091 \pm 0.046$ & $0.079 \pm 0.036$ & 0.658 & $0.109 \pm 0.062$ & $0.121 \pm 0.059$ & $0.154 \pm 0.14$ & 0.231 \\
\hline Correlation on 2 voxel distance & $0.047 \pm 0.030$ & $0.053 \pm 0.024$ & $0.056 \pm 0.027$ & 0.555 & $0.034 \pm 0.027$ & $0.036 \pm 0.023$ & $0.052 \pm 0.057$ & 0.190 \\
\hline Correlation on 3 voxel distance & $0.025 \pm 0.038$ & $0.030 \pm 0.019$ & $0.040 \pm 0.020$ & 0.231 & $0.021 \pm 0.019$ & $0.017 \pm 0.015$ & $0.027 \pm 0.030$ & 0.203 \\
\hline Contrast on 1 voxel distance & $5.30 \pm 2.23$ & $4.76 \pm 2.04$ & $4.47 \pm 1.90$ & 0.478 & $5.51 \pm 2.83$ & $4.98 \pm 3.13$ & $4.32 \pm 2.06$ & 0.492 \\
\hline Contrast on 2 voxel distance & $11.16 \pm 4.60$ & $10.45 \pm 4.24$ & $10.62 \pm 5.01$ & 0.834 & $8.13 \pm 4.25$ & $7.29 \pm 3.87$ & $6.09 \pm 2.88$ & 0.290 \\
\hline Contrast on 3 voxel distance & $14.71 \pm 6.75$ & $14.29 \pm 5.67$ & $15.48 \pm 8.17$ & 0.807 & $8.62 \pm 4.36$ & $7.94 \pm 4.19$ & $6.38 \pm 2.86$ & 0.248 \\
\hline Variance on 1 voxel distance & $0.17 \pm 0.11$ & $0.163 \pm 0.054$ & $0.129 \pm 0.029$ & 0.114 & $0.176 \pm 0.053$ & $0.185 \pm 0.052$ & $0.175 \pm 0.054$ & 0.704 \\
\hline Variance on 2 voxel distance & $0.17 \pm 0.10$ & $0.161 \pm 0.054$ & $0.129 \pm 0.032$ & 0.121 & $0.177 \pm 0.052$ & $0.186 \pm 0.051$ & $0.177 \pm 0.056$ & 0.718 \\
\hline Variance on 3 voxel distance & $0.16 \pm 0.06$ & $0.162 \pm 0.054$ & $0.131 \pm 0.038$ & 0.111 & $0.179 \pm 0.050$ & $0.188 \pm 0.051$ & $0.179 \pm 0.058$ & 0.765 \\
\hline Sum mean on 1 voxel distance & $8.83 \pm 3.47$ & $9.60 \pm 3.41$ & $11.544 \pm 3.509$ & 0.061 & $4.37 \pm 4.05$ & $4.05 \pm 1.92$ & $3.48 \pm 1.43$ & 0.351 \\
\hline Sum mean on 2 voxel distance & $8.90 \pm 3.51$ & $9.68 \pm 3.48$ & $11.354 \pm 3.407$ & 0.111 & $4.37 \pm 1.92$ & $4.02 \pm 1.87$ & $3.40 \pm 1.46$ & 0.285 \\
\hline
\end{tabular}

(Continued) 


\begin{tabular}{|c|c|c|c|c|c|c|c|c|}
\hline & \multicolumn{3}{|c|}{ Non-contrast images } & \multirow[b]{2}{*}{$p$} & \multicolumn{3}{|c|}{ Iodine-contrast images } & \multirow[b]{2}{*}{$p$} \\
\hline & One subtype & Two subtypes & $\begin{array}{c}\text { Three or four } \\
\text { subtypes }\end{array}$ & & One subtype & Two subtypes & $\begin{array}{l}\text { Three or four } \\
\text { subtypes }\end{array}$ & \\
\hline Sum mean on 3 voxel distance & $8.46 \pm 3.06$ & $9.79 \pm 3.57$ & $11.369 \pm 3.468$ & 0.051 & $4.27 \pm 1.75$ & $4.02 \pm 1.77$ & $3.36 \pm 1.44$ & 0.267 \\
\hline Inertia on 1 voxel distance & $8.37 \pm 2.23$ & $8.43 \pm 2.28$ & $10.057 \pm 1.532$ & $0.027 *$ & $6.87 \pm 1.71$ & $6.50 \pm 1.64$ & $6.85 \pm 1.71$ & 0.617 \\
\hline Inertia on 2 voxel distance & $8.45 \pm 2.26$ & $8.54 \pm 2.31$ & $10.20 \pm 1.54$ & $0.024 *$ & $6.82 \pm 1.66$ & $6.47 \pm 1.60$ & $6.82 \pm 1.70$ & 0.621 \\
\hline Inertia on 3 voxel distance & $8.39 \pm 2.26$ & $8.54 \pm 2.31$ & $10.247 \pm 1.554$ & $0.018^{*}$ & $6.70 \pm 1.56$ & $6.44 \pm 1.56$ & $6.78 \pm 1.69$ & 0.672 \\
\hline Cluster shade on 1 voxel distance & $5.30 \pm 2.23$ & $4.76 \pm 2.04$ & $4.479 \pm 1.903$ & 0.478 & $5.51 \pm 2.83$ & $4.98 \pm 3.13$ & $4.33 \pm 2.07$ & 0.492 \\
\hline Cluster shade on 2 voxel distance & $11.16 \pm 4.60$ & $10.43 \pm 4.24$ & $10.629 \pm 5.011$ & 0.834 & $8.14 \pm 4.26$ & $7.30 \pm 5.68$ & $6.10 \pm 2.88$ & 0.290 \\
\hline Cluster shade on 3 voxel distance & $14.71 \pm 6.75$ & $14.29 \pm 5.67$ & $15.480 \pm 8.177$ & 0.807 & $8.62 \pm 4.37$ & $7.941 \pm 3.87$ & $6.38 \pm 2.86$ & 0.248 \\
\hline Cluster tendency on 1 voxel distance & $-43.74 \pm 16.58$ & $-42.39 \pm 50.95$ & $-108.582 \pm 29.33$ & 0.244 & $14.25 \pm 29.92$ & $14.76 \pm 27.81$ & $3.07 \pm 20.57$ & 0.309 \\
\hline Cluster tendency on 2 voxel distance & $-37.71 \pm 86.04$ & $-36.77 \pm 116.02$ & $-82.633 \pm 04.72$ & 0.318 & $7.57 \pm 18.33$ & $8.30 \pm 16.88$ & $1.58 \pm 15.04$ & 0.374 \\
\hline Cluster tendency on 3 voxel distance & $-33.78 \pm 64.46$ & $-32.49 \pm 95.11$ & $-70.196 \pm 88.10$ & 0.314 & $7.34 \pm 15.93$ & $6.95 \pm 13.80$ & $1.56 \pm 14.53$ & 0.388 \\
\hline Homogeneity on 1 voxel distance & $4532.07 \pm 1777.15$ & $3387.64 \pm 2166.78$ & $2629.69 \pm 181.00$ & $0.025 *$ & $629.72 \pm 389.37$ & $565.77 \pm 348.42$ & $386.11 \pm 232.51$ & 0.094 \\
\hline Homogeneity on 2 voxel distance & $3480.25 \pm 1460.08$ & $2523.03 \pm 1782.39$ & $1899.41 \pm 1225.18$ & $0.023 *$ & $375.41 \pm 229.52$ & $341.68 \pm 234.28$ & $243.52 \pm 173.64$ & 0.197 \\
\hline Homogeneity on 3 voxel distance & $2895.88 \pm 1303.93$ & $2039.34 \pm 1521.84$ & $1380.43 \pm 1060.43$ & $0.008^{*}$ & $314.92 \pm 192.11$ & $289.38 \pm 191.10$ & $215.53 \pm 177.30$ & 0.273 \\
\hline Maximum probability on 1 voxel distance & $0.109 \pm 0.110$ & $0.092 \pm 0.079$ & $0.137 \pm 0.125$ & 0.248 & $0.085 \pm 0.041$ & $0.110 \pm 0.064$ & $0.116 \pm 0.067$ & 0.230 \\
\hline Maximum probability on 2 voxel distance & $0.095 \pm 0.102$ & $0.072 \pm 0.071$ & $0.117 \pm 0.123$ & 0.189 & $0.076 \pm 0.033$ & $0.095 \pm 0.055$ & $0.101 \pm 0.062$ & 0.308 \\
\hline Maximum probability on 3 voxel distance & $0.081 \pm 0.0619$ & $0.064 \pm 0.065$ & $0.107 \pm 0.120$ & 0.154 & $0.078 \pm 0.029$ & $0.092 \pm 0.051$ & $0.096 \pm 0.061$ & 0.491 \\
\hline Inverse variance on 1 voxel distance & $0.381 \pm 0.063$ & $0.402 \pm 0.045$ & $0.381 \pm 0.051$ & 0.153 & $0.413 \pm 0.050$ & $0.417 \pm 0.045$ & $0.416 \pm 0.031$ & 0.952 \\
\hline Inverse variance on 2 voxel distance & $0.316 \pm 0.060$ & $0.339 \pm 0.049$ & $0.322 \pm 0.047$ & 0.185 & $0.377 \pm 0.066$ & $0.386 \pm 0.054$ & $0.396 \pm 0.046$ & 0.598 \\
\hline Inverse variance on 3 voxel distance & $0.294 \pm 0.055$ & $0.305 \pm 0.051$ & $0.289 \pm 0.049$ & 0.487 & $0.372 \pm 0.059$ & $0.375 \pm 0.056$ & $0.390 \pm 0.047$ & 0.558 \\
\hline
\end{tabular}

Features showing uniform tendency are expressed in bold font. $* \mathrm{P}<0.05$.

In addition, radiomic parameters might be useful for noninvasive in vivo monitoring of longitudinal changes in tumor heterogeneity. Lung cancer cells within tumors show homogeneous cell populations until relatively late in tumor progression, when hyperproliferation and increased genetic instability result in distinct clonal subpopulations $[18,19]$. Several studies have shown that mechanisms of acquired drug resistance to EGFR inhibitors are related to several genotypic and phenotypic changes [27, 28]. Using a radiogenomic approach to quantify tumor heterogeneity on images may allow longitudinal studies of clonal evolution during treatment as well.

Our study had several limitations. First, the proportion of the tumor with three or four mixtures of histologic subtypes was relatively small. The relationship of the quantitative radiomic variables and pathologic heterogeneity and the values of the quantitative radiomic variables may be influenced by the sample size. Second, all cases were collected from a single institute. Larger prospective studies from multiple centers are needed. Third, we excluded patients with variant subtypes including mucinous pattern. However, we decided to exclude this subtype because reports regarding the survival of mucinous lung adenocarcinomas are limited $[29,30]$.

In conclusion, various radiomic variables from DECT of lung adenocarcinoma reflect pathologic intratumoral heterogeneity, which may be a helpful predictor of intratumoral heterogeneity of the whole tumor, considering that current genomic analyses are limited by the fact that they rely on a single tumor biopsy.

\section{MATERIALS AND METHODS}

\section{Patients}

This study was performed as part of an ongoing prospective clinical trial aimed at determining the value of imaging biomarkers for the prediction of tumor aggressiveness and prognosis in patient with operable lung adenocarcinoma (NCT01482585). This study was approved by the institutional review board (SMC 201109-083) and written informed consent was obtained. 
From November 2011 to December 2012, a total of 92 patients with operable lung adenocarcinoma were eligible for our study. The inclusion criteria of our study were as follows: (1) Clinically and radiologically suspected lung adenocarcinoma, (2) Newly diagnosed stage I or II disease from clinical work-up including F-18fluorodeoxyglucose (FDG) positron emission tomography (PET)/CT, (3) Eastern Cooperative Oncology Group (ECOG) performance status of 0 or 1 and eligible for surgery, (4) Age 20 years or older, (5) Able to tolerate DECT imaging as required per protocol, and (6) Able to give study-specific informed consent. The exclusion criteria were: (1) Prior malignancy, (2) Scheduled for definitive radiation therapy or neoadjuvant concurrent chemoradiation therapy, and (3) Poor cardiopulmonary reserve (a contraindication for surgery).

\section{CT imaging and analysis}

Patients underwent CT examination using a dualsource CT scanner (Somatom Definition Flash; Siemens Medical Solutions, Forchheim, Germany) with the dualenergy technique. Three types of data set were generated from the DECT scanning: $80 \mathrm{kV}, 140 \mathrm{kV}$, and enhanced weighted-average images. See the supplement and Supplementary Figure S2 for further details about the image acquisition protocol and reconstruction process.

Virtual non-enhanced images and iodine-enhanced images were generated using the liver Virtual NonContrast (VNC) application node of dedicated dual-energy post-processing software (Syngo Dual Energy; Siemens Medical Solution, Forchheim, Germany). To obtain the iodine value of both the solid and ground-glass opacity (GGO) component in each tumor, post-processing was performed with two different types of software. Image data were reconstructed with a section thickness of $1 \mathrm{~mm}$ using a D30f (medium smooth) kernel for the iodineenhanced image and a D45f (medium smooth) kernel for the virtual non-enhanced image.

In the quantitative analysis, regions of interest (ROIs) were delineated on the axial images to generate a volume of interest which included the entire tumor. Initially, we assessed the stability of all 51 radiomic parameters for which we performed the concordance correlation coefficients (CCC) regarding radiomic values extracted from two ROIs drawn by two radiologists in 25 randomly selected patients. From the stability test, we found that all radiomic parameters were stable, where all CCCs were very high or high (range, 0.888 - 0.999; mean, 0.934; SD, 0.008). As a next step, all included patient were handled by one radiologist.

Fifty one quantitative radiomic features were derived from this ROI to evaluate the heterogeneity of the tumors (Figure 2). The physical features included volume, size, density and mass. The histogram features included skewness, kurtosis, and Hounsfield units (HU) at the $2.5^{\text {th }}, 25^{\text {th }}, 50^{\text {th }}, 75^{\text {th }}$ and $97.5^{\text {th }}$ percentiles. The regional features included uniformity, entropy, intensity variability and size-zone variability. There were 36 local texture features, including energy, entropy, correlation, contrast, variance, sum mean, inertia, cluster shade, cluster tendency, homogeneity, maximum probability, and inverse variance. Each local texture feature was derived from 13 directions according to 1-voxel, 2-voxel, and 3-voxel distances at the gray value for each voxel. These radiomic features were evaluated both on non-contrast images and iodine contrast images. See the supplement for further details regarding the extraction of quantitative radiomic parameters.

\section{Pathologic evaluation and analysis}

For tumor sampling, tumor tissues with an interval of per approximately $10 \mathrm{~mm}$ were taken from the tumor specimen and placed on a slide. All slides were scanned to produce a high- resolution digital image $(0.25 \mu \mathrm{m} / \mathrm{pixel}$ at $40 \times$ magnification) using the Aperio Slide Scanning System (ScanScope T3; Aperio Technologies Inc., Vista, CA, USA). Two experienced lung pathologists with 13 and 18 years of experience (J.Y.J., Y.C.) interpreted all tissue sections by virtual slides using ImageScope viewing software (Aperio Technologies, Inc.) and a high-resolution monitor. For each case, the specimens were reviewed according to the International Association for the Study of Lung Cancer (IASLC), the American Thoracic Society (ATS), and the European Respiratory Society (ERS) International Multidisciplinary Lung Adenocarcinoma Classification Criteria [3] and comprehensive histologic subtyping was performed for a whole primary tumor in a semi-quantitative manner. The extent of existent tumor histologic subtypes and central fibrosis was quantified to the nearest $5 \%$ level, adding up to a total of $100 \%$ of the subtype components per tumor. They reported each of tumors as a relative ratio among total $100 \%$ in terms of all five with its whole histologic subtype.

The pathologic heterogeneity index was calculated from each tumor to evaluate the degree of pathologic heterogeneity, which was confirmed to reflect the survival predictive value according to the proportion of mixed histologic subtypes that were derived from the hazard ratio (HR) of each subtype using the disease-free survival curve of a large-scale study [4]. Further details about the pathologic heterogeneity index are described in the supplement.

\section{Statistical analysis}

A multivariate logistic regression model with the stepwise variable selection procedure based on Akaike's Information Criterion (AIC) was applied to validate the performance of selected radiomic features to predict actually pathologic heterogeneity. Ten-fold cross- 
validation [31] was used to evaluate the performance of this prediction model. After then, Pearson correlation analysis was applied to compare the predictive values from made prediction model with pathologic heterogeneity in each. The Spearman correlation coefficient was used to determine the relationship between radiomic features and pathologic heterogeneity index. When statistically significant, an absolute Rho ( $\rho$ ) value between 0.00 and 0.19 was considered very weak correlation, $0.20-$ 0.39, weak, 0.40-0.59, moderate, 0.60-0.79 strong and greater than 0.80 , strong [32]. Radiomic parameters were compared among the three different groups that had been classified according to the number of histologic subtype within a tumor (e.g., tumor consistent with a single histologic subtype, two histologic subtypes, or three or four histologic subtypes) using ANOVA with Tukey's post hoc test. In the cases with multiple tumors in a patient, we did not take into account within-patient correlation because each tumor was considered an independent synchronous lesion [33].

Statistical significance was evaluated with software (SPSS, version 19.0, 2010; SPSS, Chicago, Ill). A P value less than 0.05 was considered to represent a statistically significant difference.

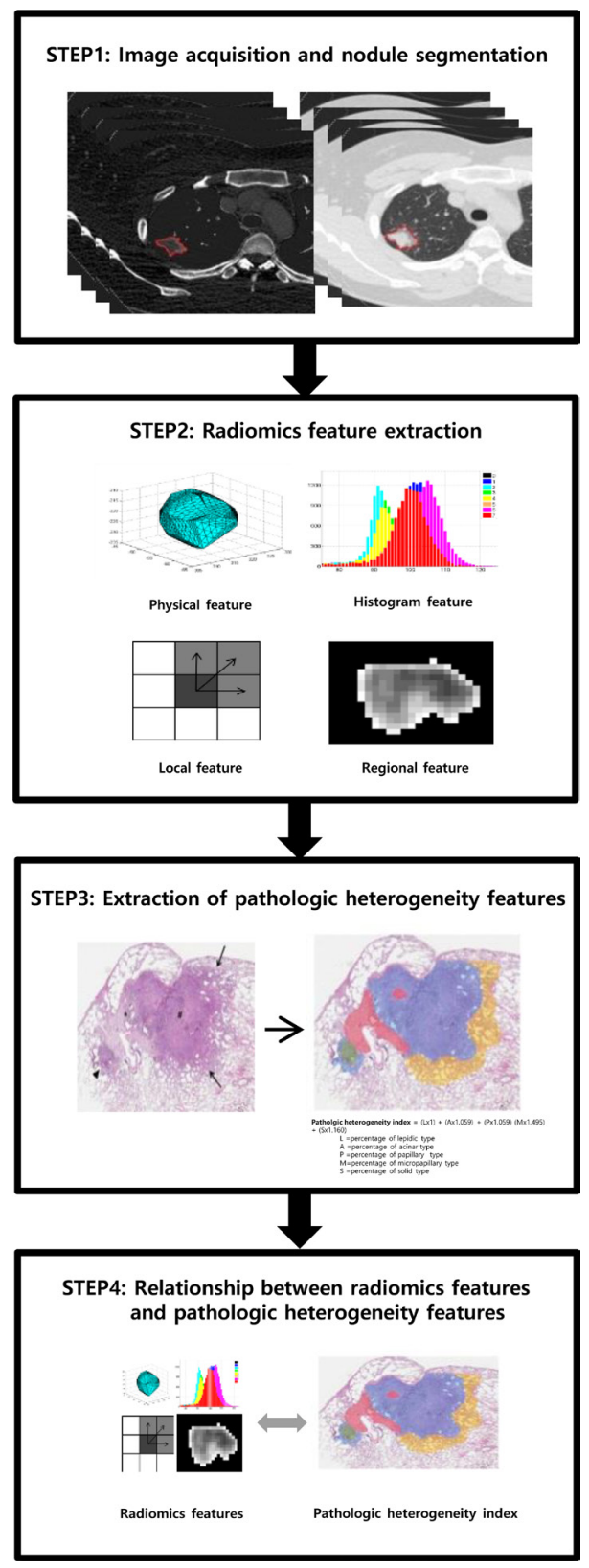

Figure 2: Radiomic data extraction and analysis workflow. 


\section{ACKNOWLEDGMENTS}

None.

\section{CONFLICTS OF INTEREST}

The authors declare no conflicts of interest.

\section{GRANT SUPPORT}

This study was supported by a grant from the Korean Foundation for Cancer Research (KFCR-CB-2011-02-02).

\section{REFERENCES}

1. Siegel R, Naishadham D, Jemal A. Cancer statistics, 2013. CA Cancer J Clin. 2013; 63:11-30.

2. Curado MP, International Agency for Research on $\mathrm{C}$ and International Association of Cancer R. Cancer incidence in five continents, Volume IX. Lyon; Geneva: International Agency for Research on Cancer ; Distributed by WHO Press, World Health Organization; 2008.

3. Travis WD, Brambilla E, Noguchi M, Nicholson AG, Geisinger KR, Yatabe Y, Beer DG, Powell CA, Riely GJ, Van Schil PE, Garg K, Austin JH, Asamura H, et al. International association for the study of lung cancer/ american thoracic society/european respiratory society international multidisciplinary classification of lung adenocarcinoma. J Thorac Oncol. 2011; 6:244-285.

4. Hung JJ, Yeh YC, Jeng WJ, Wu KJ, Huang BS, Wu YC, Chou TY, Hsu WH. Predictive value of the international association for the study of lung cancer/American Thoracic Society/European Respiratory Society classification of lung adenocarcinoma in tumor recurrence and patient survival. J Clin Oncol. 2014; 32:2357-2364.

5. Campos-Parra AD, Aviles A, Contreras-Reyes S, RojasMarin CE, Sanchez-Reyes R, Borbolla-Escoboza RJ, Arrieta O. Relevance of the novel IASLC/ATS/ERS classification of lung adenocarcinoma in advanced disease. Eur Respir J. 2014; 43:1439-1447.

6. Motoi N, Szoke J, Riely GJ, Seshan VE, Kris MG, Rusch VW, Gerald WL, Travis WD. Lung adenocarcinoma: modification of the 2004 WHO mixed subtype to include the major histologic subtype suggests correlations between papillary and micropapillary adenocarcinoma subtypes, EGFR mutations and gene expression analysis. Am J Surg Pathol. 2008; 32:810-827.

7. Kadota K, Colovos C, Suzuki K, Rizk NP, Dunphy MP, Zabor EC, Sima CS, Yoshizawa A, Travis WD, Rusch VW, Adusumilli PS. FDG-PET SUVmax combined with IASLC/ ATS/ERS histologic classification improves the prognostic stratification of patients with stage I lung adenocarcinoma. Ann Surg Oncol. 2012; 19:3598-3605.
8. Lee HY, Jeong JY, Lee KS, Kim HJ, Han J, Kim BT, Kim J, Shim YM, Kim JH, Song I. Solitary pulmonary nodular lung adenocarcinoma: correlation of histopathologic scoring and patient survival with imaging biomarkers. Radiology. 2012; 264:884-893.

9. Sica G, Yoshizawa A, Sima CS, Azzoli CG, Downey RJ, Rusch VW, Travis WD, Moreira AL. A grading system of lung adenocarcinomas based on histologic pattern is predictive of disease recurrence in stage I tumors. Am J Surg Pathol. 2010; 34:1155-1162.

10. Win T, Miles KA, Janes SM, Ganeshan B, Shastry M, Endozo R, Meagher M, Shortman RI, Wan S, Kayani I, Ell PJ, Groves AM. Tumor heterogeneity and permeability as measured on the CT component of PET/CT predict survival in patients with non-small cell lung cancer. Clin Cancer Res. 2013; 19:3591-3599.

11. Ganeshan B, Panayiotou E, Burnand K, Dizdarevic S, Miles $\mathrm{K}$. Tumour heterogeneity in non-small cell lung carcinoma assessed by CT texture analysis: a potential marker of survival. Eur Radiol. 2012; 22:796-802.

12. O'Connor JP, Rose CJ, Waterton JC, Carano RA, Parker GJ, Jackson A. Imaging intratumor heterogeneity: role in therapy response, resistance, and clinical outcome. Clin Cancer Res. 2015; 21:249-257.

13. Jamal-Hanjani M, Quezada SA, Larkin J, Swanton C. Translational implications of tumor heterogeneity. Clin Cancer Res. 2015; 21:1258-1266.

14. Gerlinger M, Rowan AJ, Horswell S, Larkin J, Endesfelder D, Gronroos E, Martinez P, Matthews N, Stewart A, Tarpey P, Varela I, Phillimore B, Begum S, et al. Intratumor heterogeneity and branched evolution revealed by multiregion sequencing. N Engl J Med. 2012; 366:883-892.

15. Meacham CE, Morrison SJ. Tumour heterogeneity and cancer cell plasticity. Nature. 2013; 501:328-337.

16. Ding L, Ley TJ, Larson DE, Miller CA, Koboldt DC, Welch JS, Ritchey JK, Young MA, Lamprecht T, McLellan MD, McMichael JF, Wallis JW, Lu C, et al. Clonal evolution in relapsed acute myeloid leukaemia revealed by wholegenome sequencing. Nature. 2012; 481:506-510.

17. Greaves M, Maley CC. Clonal evolution in cancer. Nature. 2012; 481:306-313.

18. Zhang J, Fujimoto J, Zhang J, Wedge DC, Song X, Zhang J, Seth S, Chow CW, Cao Y, Gumbs C, Gold KA, Kalhor $\mathrm{N}$, Little L, et al. Intratumor heterogeneity in localized lung adenocarcinomas delineated by multiregion sequencing. Science. 2014; 346:256-259.

19. de Bruin EC, McGranahan N, Mitter R, Salm M, Wedge DC, Yates L, Jamal-Hanjani M, Shafi S, Murugaesu N, Rowan AJ, Gronroos E, Muhammad MA, Horswell S, et al. Spatial and temporal diversity in genomic instability processes defines lung cancer evolution. Science. 2014; 346:251-256. 
20. Hanahan D, Weinberg RA. Hallmarks of cancer: the next generation. Cell. 2011; 144:646-674.

21. Ganeshan B, Goh V, Mandeville HC, Ng QS, Hoskin PJ, Miles KA. Non-small cell lung cancer: histopathologic correlates for texture parameters at CT. Radiology. 2013; 266:326-336

22. Tixier F, Hatt M, Le Rest CC, Le Pogam A, Corcos L, Visvikis D. Reproducibility of tumor uptake heterogeneity characterization through textural feature analysis in $18 \mathrm{~F}$ FDG PET. J Nucl Med. 2012; 53:693-700.

23. Willaime JM, Turkheimer FE, Kenny LM, Aboagye EO. Quantification of intra-tumour cell proliferation heterogeneity using imaging descriptors of $18 \mathrm{~F}$ fluorothymidine-positron emission tomography. Phys Med Biol. 2013; 58:187-203.

24. Alic L, Niessen WJ, Veenland JF. Quantification of heterogeneity as a biomarker in tumor imaging: a systematic review. PLoS One. 2014; 9:e110300.

25. Aerts HJ, Velazquez ER, Leijenaar RT, Parmar C, Grossmann P, Carvalho S, Bussink J, Monshouwer R, Haibe-Kains B, Rietveld D, Hoebers F, Rietbergen MM, Leemans CR, Dekker A, Quackenbush J, Gillies RJ, Lambin P. Decoding tumour phenotype by noninvasive imaging using a quantitative radiomics approach. Nat Commun. 2014; 5:4006.

26. Ganeshan B, Abaleke S, Young RC, Chatwin CR, Miles KA. Texture analysis of non-small cell lung cancer on unenhanced computed tomography: initial evidence for a relationship with tumour glucose metabolism and stage. Cancer Imaging. 2010; 10:137-143.

27. Sequist LV, Waltman BA, Dias-Santagata D, Digumarthy S, Turke AB, Fidias P, Bergethon K, Shaw AT, Gettinger
S, Cosper AK, Akhavanfard S, Heist RS, Temel J, et al. Genotypic and Histological Evolution of Lung Cancers Acquiring Resistance to EGFR Inhibitors. Science Translational Medicine. 2011; 3:75ra26-75ra26.

28. Bai H, Wang Z, Chen K, Zhao J, Lee JJ, Wang S, Zhou Q, Zhuo M, Mao L, An T, Duan J, Yang L, Wu M, Liang Z, Wang Y, Kang X, Wang J. Influence of chemotherapy on EGFR mutation status among patients with non-small-cell lung cancer. J Clin Oncol. 2012; 30:3077-3083.

29. Ichinokawa H, Ishii G, Nagai K, Yoshida J, Nishimura M, Hishida T, Suzuki K, Ochiai A. Clinicopathological characteristics of primary lung adenocarcinoma predominantly composed of goblet cells in surgically resected cases. Pathol Int. 2011; 61:423-429.

30. Warth A, Muley T, Meister M, Stenzinger A, Thomas M, Schirmacher P, Schnabel PA, Budczies J, Hoffmann H, Weichert W. The novel histologic International Association for the Study of Lung Cancer/American Thoracic Society/ European Respiratory Society classification system of lung adenocarcinoma is a stage-independent predictor of survival. J Clin Oncol. 2012; 30:1438-1446.

31. Simon RM, Subramanian J, Li M-C, Menezes S. Using cross-validation to evaluate predictive accuracy of survival risk classifiers based on high-dimensional data. Briefings in bioinformatics. 2011; 12:203-214.

32. Taylor R. Interpretation of the correlation coefficient: a basic review. Journal of diagnostic medical sonography. 1990; 6:35-39.

33. Kim HK, Choi YS, Kim K, Shim YM, Jeong SY, Lee KS, Kwon OJ, Kim J. Management of ground-glass opacity lesions detected in patients with otherwise operable nonsmall cell lung cancer. J Thorac Oncol. 2009; 4:1242-1246. 